\title{
A NOTE ON THE REPRESENTATION THEORY OF THE HECKE ALGEBRA OF TYPE $F_{4}$ \\ by C. A. PALLIKAROS
}

(Received 18 July, 1995)

Introduction. In [4] Dipper and James investigated the representation theory of Hecke algebras of type $B_{n}, H\left(B_{n}\right)$. Using the results in [4] and exploiting the fact that the Hecke algebra of type $F_{4}$, denoted by $H(W)$, contains two copies of $H\left(B_{3}\right)$ certain right ideals of $H(W)$ will be constructed in this paper. These right ideals will be proved to be irreducible on the assumption that $H(W)$ is semisimple.

$H(W)$ will be defined as a vector space over a field $K$ of arbitrary characteristic and its definition will depend on $q$ and $Q$ where $q, Q \in K$. From the right ideals constructed in this paper we will get some 8-dimensional and some 9-dimensional matrix representations of $H(W)$. The characters of these representations agree with the characters given in Geck [5]. In [5], Geck calculated the characters of the generic Hecke algebra of type $F_{4}$ by constructing matrix representations of degree at most 8 . His representing matrices for the generators involve denominators. The method used in this paper to construct representations is different from Geck's and the representing matrices obtained here do not involve any denominators.

In the special case where char $K=0$ and $q=1=Q$, the right ideals constructed in this paper will give all the 8-dimensional and 9-dimensional ordinary irreducible representations of the Weyl group of type $F_{4}$, the character table of which is given in $[1$, p. 413].

1. The Weyl group of type $\boldsymbol{F}_{\mathbf{4}}$. The Weyl group of type $F_{4}$, which we denote by $W$, is generated by $t_{2}, t, s_{1}, s_{2}$ where

$$
t_{2} s_{i}=s_{i} t_{2} \text { for } i=1,2, \quad t s_{2}=s_{2} t \text { and }\left(t_{2} t\right)^{3}=1=\left(s_{1} s_{2}\right)^{3}=\left(t s_{1}\right)^{4} \text {. }
$$

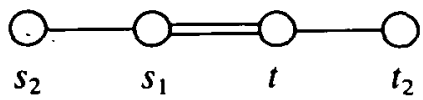

In particular $W$ has order 1152 and has two parabolic subgroups which are isomorphic to the Weyl group of type $B_{3}$, namely $W_{1}$ which is generated by $\left\{t, s_{1}, s_{2}\right\}$ and $W_{2}$ which is generated by $\left\{s_{1}, t, t_{2}\right\}$.

1.1. Remark. It is easy to see that there exists an automorphism $\rho$ of $W$ where $\rho\left(s_{1}\right)=t, \rho(t)=s_{1}, \rho\left(s_{2}\right)=t_{2}$ and $\rho\left(t_{2}\right)=s_{2}$.

1.2. Definition. Each element $w$ of $W$ is a product of the generators $s_{1}, s_{2}, t, t_{2}$. The length of $w, \ell(w)$, is defined to be the minimal length of any expression for $w$ in this form. Hence if $w \in W$, then $w=v_{1} v_{2} \ldots v_{\ell}$ where each $v_{i}$ belongs to $\left\{s_{2}, s_{1}, t, t_{2}\right\}$ and $\ell=\ell(w)$. Such an expression for $w$ is called a reduced expression.

1.3. Definition. If $H$ is a subgroup of a group $G$, we call $H g$ a right coset of $H$ in $G$.

Glasgow Math. J. 39 (1997) 43-50. 
If $\ell(h g)=\ell(h)+\ell(g)$ for all $h \in H$, we say that $g$ is a distinguished right coset representative of $H$ in $G$. Distinguished and coset representatives are defined in a similar way.

1.4. Lemma. The set $S$ given below is a complete set of distinguished right coset representatives of $W_{1}$ in $W$.

$$
\begin{aligned}
S=\left\{s^{*}=\right. & t_{2} t s_{1} t t_{2} s_{2} s_{1} t s_{1} s_{2} t_{2} t s_{1} t t_{2}, t_{2} t s_{1} t t_{2} s_{2} s_{1} t s_{1} s_{2} t_{2} t s_{1} t, t_{2} t s_{1} t t_{2} s_{2} s_{1} t s_{1} s_{2} t_{2} t s_{1}, \\
& t_{2} t s_{1} t t_{2} s_{2} s_{1} t s_{1} s_{2} t_{2} t, t_{2} t s_{1} t t_{2} s_{2} s_{1} t s_{1} t_{2} t, t_{2} t s_{1} t t_{2} s_{2} s_{1} t s_{1} s_{2} t_{2}, t_{2} t s_{1} t t_{2} s_{2} s_{1} t s_{1} s_{2}, \\
& t_{2} t s_{1} t t_{2} s_{2} s_{1} t s_{1} t_{2}, t_{2} t s_{1} t t_{2} s_{2} s_{1} t s_{1}, t_{2} t s_{1} t t_{2} s_{2} s_{1} t t_{2}, t_{2} t s_{1} t s_{2} s_{1} t t_{2}, t_{2} t s_{1} t s_{2} s_{1} t, \\
& t_{2} t s_{1} t t_{2} s_{2} s_{1} t, t_{2} t s_{1} t t_{2} s_{2} s_{1}, t_{2} t s_{1} t t_{2} s_{2}, t_{2} t s_{1} t s_{2} s_{1}, t_{2} t s_{1} t t_{2}, t_{2} t s_{1} t s_{2}, t_{2} t s_{1} t, \\
& \left.t_{2} t s_{1} s_{2}, t_{2} t s_{1}, t_{2} t, t_{2}, 1\right\} .
\end{aligned}
$$

Proof. First note that $u=t s_{1} t s_{1} s_{2} s_{1} t s_{1} s_{2} t_{2} t s_{1} t t_{2} s_{2} s_{1} t s_{1} s_{2} t_{2} t s_{1} t t_{2}$ is the longest element in $W$ with $\ell(u)=24$, hence the expression given above for $u$ is a reduced expression. Note also that $v=t s_{1} t s_{1} s_{2} s_{1} t s_{1} s_{2}$ is the longest element in $W_{1}$. The proof of the lemma now follows easily once we notice that $u=v s^{*}$.

1.5. Remark. In $[4,2.2]$, a canonical way for listing the elements of $W_{1}$ is given. Combining this result with Lemma 1.4, we get a canonical way for listing the elements of $W$ in reduced form.

1.6. Remark. A complete set of distinguished left coset representatives of $W_{1}$ in $W$ can easily be deduced from symmetry. Moreover, using the automorphism $\rho$ of $W$ given in Remark 1.1, we can easily find complete sets of distinguished right or left coset representatives of $W_{2}$ in $W$.

\section{The Hecke algebra of type $F_{4}$.}

2.1. Definition. Let $K$ be a field and let $q$ and $Q$ be non-zero elements of $K$. The Hecke algebra of type $F_{4}, H(W)$, over $K$ with respect to $q$ and $Q$ is defined to be a vector space over $K$ with basis $\left\{T_{w}: w \in W\right\}$ with the following multiplication structure:

$T_{1}$ is the multiplicative identity of $H$;

$T_{x} T_{x}=q+(q-1) T_{x}$ if $x \in\left\{s_{1}, s_{2}\right\}$

$T_{y} T_{y}=Q+(Q-1) T_{y}$ if $y \in\left\{t, t_{2}\right\}$

if $w=v_{1} v_{2} \ldots v_{\ell}$ is a reduced expression for $w \in W$ where each $v_{i}$ belongs to $\left\{s_{2}, s_{1}, t, t_{2}\right\}$, then $T_{w}=T_{v_{1}} T_{v_{2}} \ldots T_{v_{\ell}}$.

(Here and later we write $r$ to denote $r T_{1}$ where $r \in K$.)

2.2. Remarks.

(i) Alternatively, the multiplication structure of $H(W)$ can be defined using the defining relations given for example in [5, p. 51].

(ii) If we set $q=Q=1$ in Definition 2.1 we recover the group algebra of $W$.

2.3. Remark. We can form the Hecke algebras $H\left(W_{1}\right)$ and $H\left(W_{2}\right)$ in the obvious way. Then $H\left(W_{1}\right)$ and $H\left(W_{2}\right)$ are subalgebras of $H(W)$ and they are both isomorphic to the Hecke algebra of type $B_{3}$. The representation theory of Hecke algebras of type $B_{n}$ is given in [4]. 
2.4. Remark. There is an automorphism $\theta$ of $H(W)$, the action of which on the generators is given by

$$
\begin{array}{ll}
\theta\left(T_{x}\right)=(q-1)-T_{x}, & x \in\left\{s_{1}, s_{2}\right\}, \\
\theta\left(T_{y}\right)=(Q-1)-T_{y}, & y \in\left\{t, t_{2}\right\} .
\end{array}
$$

The ordinary irreducible representations of $W_{1}$ (and of $W_{2}$ ) are indexed by pairs of partitions $(\alpha)(\beta)$ where $\alpha$ is a partition of $a, \beta$ is a partition of $b(a+b=3)$. $(\ln [4,5.6]$ it is shown that the ordinary irreducible representations of $H\left(B_{3}\right)$ are indexed in the same way.) We therefore have that the ordinary irreducible representations of $W_{1}$ are indexed by

$$
\begin{aligned}
& \phi_{1}:(3)(0), \phi_{2}:(21)(0), \phi_{3}:\left(1^{3}\right)(0), \phi_{4}:(2)(1), \phi_{5}:\left(1^{2}\right)(1), \\
& \phi_{6}:(1)(2), \phi_{7}:(1)\left(1^{2}\right), \phi_{8}:(0)(3), \phi_{9}:(0)(21), \phi_{10}:(0)\left(1^{3}\right)
\end{aligned}
$$

where for $i=1, \ldots, 10, \phi_{i}$ denotes the character of the irreducible representation which corresponds to the given partition.

Let $\hat{\phi}_{i}(1 \leq i \leq 10)$ be a representation of $W_{1}$ with character $\phi_{i}$. We can then find a representation $\hat{\psi}_{i}$ of $W_{2}$ where $\hat{\psi}_{i}\left(s_{1}\right)=\hat{\phi}_{i}(t), \hat{\psi}_{i}(t)=\hat{\phi}_{i}\left(s_{1}\right), \hat{\psi}_{i}\left(t_{2}\right)=\hat{\phi}_{i}\left(s_{2}\right)$. Let $\psi_{i}$ be the character of the representation $\hat{\psi}_{i}$. Then $\psi_{i}, 1 \leq i \leq 10$, give all the ordinary irreducible characters of $W_{2}$. We will need the right ideals of $H\left(W_{1}\right)$ with generators

$$
\begin{aligned}
& x_{3}=\left(1+T_{t}\right)\left(q+T_{s_{1} t s_{1}}\right)\left(q^{2}+T_{s_{2} s_{1} s_{1} s_{2}}\right)\left(1-\frac{1}{q} T_{s_{1}}-\frac{1}{q} T_{s_{2}}+\frac{1}{q^{2}} T_{s_{1} s_{2}}+\frac{1}{q^{2}} T_{s_{2} s_{1}}-\frac{1}{q^{3}} T_{s_{1} s_{2} s_{1}}\right), \\
& x_{4}=\left(1+T_{t}\right)\left(q+T_{s_{1} t s_{1}}\right) T_{s_{2} s_{1}}\left(Q-T_{t}\right)\left(1+T_{s_{2}}\right), \\
& x_{5}=\left(1+T_{t}\right)\left(q+T_{s_{1} t s_{1}}\right) T_{s_{2} s_{1}}\left(Q-T_{t}\right)\left(q-T_{s_{2}}\right), \\
& x_{6}=\left(1+T_{t}\right) T_{s_{1} s_{2}}\left(Q-T_{t}\right)\left(Q q-T_{s_{1} s_{1}}\right)\left(1+T_{s_{1}}\right), \\
& x_{7}=\left(1+T_{t}\right) T_{s_{1} s_{2}}\left(Q-T_{t}\right)\left(Q q-T_{s_{1} s_{1}}\right)\left(q-T_{s_{1}}\right), \\
& x_{8}=\left(Q-T_{t}\right)\left(Q q-T_{s_{1} t s_{1}}\right)\left(Q q^{2}-T_{s_{2} s_{1} t s_{1} s_{2}}\right)\left(1+T_{s_{1}}+T_{s_{2}}+T_{s_{1} s_{2}}+T_{s_{2} s_{1}}+T_{s_{1} s_{2} s_{1}}\right)
\end{aligned}
$$

and the right ideals of $H\left(W_{2}\right)$ with generators

$$
\begin{aligned}
& y_{1}=\left(1+T_{s_{1}}\right)\left(Q+T_{t s_{1} t}\right)\left(Q^{2}+T_{t_{2} s_{1} t_{2}}\right)\left(1+T_{t}+T_{t_{2}}+T_{t t_{2}}+T_{t_{2} t}+T_{t t_{2} t}\right) \text {, } \\
& y_{3}=\left(1+T_{s_{1}}\right)\left(Q+T_{t s_{1}}\right)\left(Q^{2}+T_{t_{2} s_{1} t_{2}}\right)\left(1-\frac{1}{Q} T_{t}-\frac{1}{Q} T_{t_{2}}+\frac{1}{Q^{2}} T_{t t_{2}}+\frac{1}{Q^{2}} T_{t_{2} t}-\frac{1}{Q^{3}} T_{t t_{2}}\right), \\
& y_{4}=\left(1+T_{s_{1}}\right)\left(Q+T_{t s, t}\right) T_{t_{2} t}\left(q-T_{s_{1}}\right)\left(1+T_{t_{2}}\right) \text {, } \\
& y_{7}=\left(1+T_{s_{1}}\right) T_{t t_{2}}\left(q-T_{s_{1}}\right)\left(Q q-T_{t s_{1}}\right)\left(Q-T_{t}\right) \text {, } \\
& y_{8}=\left(q-T_{s_{1}}\right)\left(Q q-T_{t s_{1} t}\right)\left(Q^{2} q-T_{t_{2} t s_{1} t_{2}}\right)\left(1+T_{t}+T_{t_{2}}+T_{t t_{2}}+T_{t_{2} t}+T_{t_{2} t}\right) \text {, } \\
& y_{9}=\left(q-T_{s_{1}}\right)\left(Q q-T_{t s_{1}}\right)\left(Q^{2} q-T_{t_{2} t_{1} t t_{2}}\right)\left(1+T_{t}\right) T_{t_{2}}\left(Q-T_{t}\right) \text {, } \\
& y_{10}=\left(q-T_{s_{1}}\right)\left(Q q-T_{t s_{1} t}\right)\left(Q^{2} q-T_{t_{2} s_{1} t_{2}}\right)\left(1-\frac{1}{Q} T_{t}-\frac{1}{Q} T_{t_{2}}+\frac{1}{Q^{2}} T_{t t_{2}}+\frac{1}{Q^{2}} T_{t_{2} t}-\frac{1}{Q^{3}} T_{t_{2} t}\right) \text {. }
\end{aligned}
$$

2.5. Remark. The method of constructing the generators $x_{i}$ and $y_{j}$ given above is described in $[4,5.7]$ and these generators were constructed so that in the special case 
char $K=0$ and $q=Q=1, x_{i} H\left(W_{1}\right)$ gives a representation for $W_{1}$ with character $\phi_{i}$ $(i=3,4,5,6,7,8)$ and $y_{j} H\left(W_{2}\right)$ gives a representation of $W_{2}$ with character $\psi_{j}$ $(j=1,3,4,7,8,9,10)$.

2.6. REMARK. The ordinary character table of $W$, the Weyl group of type $F_{4}$, is given in $[1, p .413]$. We will refer to this table for the labelling of the characters (that is the $i$ th character, $\chi_{i}$, in our notation is the $i$ th character in that table). Inducing up some of the characters of $W_{1}$ and $W_{2}$ we get the following table:

\begin{tabular}{|c|r|c|}
\hline$i$ & $j$ & $\begin{array}{r}\text { The unique common constituent } \\
\text { of } \phi_{i} \uparrow w_{1}^{W} \text { and } \psi_{j} \uparrow w_{2}\end{array}$ \\
\hline 6 & 1 & $\chi_{10}$ \\
7 & 8 & $\chi_{11}$ \\
8 & 7 & $\chi_{12}$ \\
5 & 10 & $\chi_{13}$ \\
4 & 8 & $\chi_{21}$ \\
7 & 3 & $\chi_{22}$ \\
8 & 4 & $\chi_{23}$ \\
3 & 7 & $\chi_{24}$ \\
6 & 9 & $\chi_{25}$ \\
\hline
\end{tabular}

In the next section we will make use of these results to construct certain right ideals of $H(W)$ which will be proved to be irreducible on the assumption that $H(W)$ is semisimple. In the special case char $K=0, q=Q=1$, the representations of $W$ corresponding to these right ideals have characters $\chi_{m}$ for $10 \leq m \leq 13$ and $21 \leq m \leq 24$ (that is, we will get all the 8-dimensional and all the 9-dimensional ordinary irreducible representations of $W$ ).

3. Certain right ideals of $\boldsymbol{H}(\boldsymbol{W})$. In this section we denote $H(W)$ by $H$. In what follows we will need the following preliminary lemma the proof of which is standard.

3.1. Lemma. Suppose $e, f \in H$ where $e^{2}=k e(k \in K, k \neq 0)$. Then $\operatorname{Hom}_{H}(e H, f H) \cong$ fHe as vector spaces over $K$.

Proof. The proof is easy once we notice that any $H$-homomorphism from $e H$ to $f H$ is given by left multiplication with fhe for some $h \in H$.

3.2. Corollary. In addition to the hypothesis of Lemma 3.1, assume that $H$ is semisimple and that fHe is 1-dimensional as a vector space over $K$. Then we can conclude that $(\mathrm{fHe}) \mathrm{H}$ is a simple $H$-module.

Proof. In view of Lemma 3.1 we can deduce that $\operatorname{Hom}_{H}(e H, f H)$ is 1-dimensional as a vector space over $K$. It now follows from the fact that $H$ is assumed to be semisimple that $e H$ and $f H$ have exactly one common composition factor. Now $f H e \neq 0$ by assumption, so we can find $h^{*} \in H$ such that $f h^{*} e \neq 0$. Since fHe is 1-dimensional we get that $\left(f h^{*} e\right) H=(f H e) H$. Defining $\alpha: e H \rightarrow f H$ such that $e h \mapsto\left(f h^{*} e\right) h(h \in H)$, we get that 
$\operatorname{Im} \alpha=\left(f h^{*} e\right) H \neq 0$ and that every composition factor of $\left(f h^{*} e\right) H$ is a composition factor of $f H$ and of $e H$.

It follows that the unique common composition factor of $e H$ and of $f H$ is isomorphic to $\left(f h^{*} e\right) H$. Hence $(f H e) H=(f h * e) H$ is simple.

For the remainder of this section assume that $H$ is semisimple.

Define $P(Q, q)$, the Poincare polynomial for $H(W)$, by $\left(\sum_{w \in W} T_{w}\right)^{2}=P(Q, q)\left(\sum_{w \in W} T_{w^{\prime}}\right)$. It follows from the assumption that $H$ is semisimple that $P(Q, q) \neq 0$. In particular $(1+q)(1+Q) \neq 0$ since $(1+q)(1+Q)$ divides $P(Q, q)$.

We are now going to use Corollary 3.2 in order to construct a right ideal of $H$ which will turn out to be a simple $H$-module. Let $f=x_{7}$ and $e=y_{8}$. We consider fHe as a vector space over $K$. First note that $e^{2}=k e$ where

$$
k=Q^{3}(1+q)(1+Q)\left(1+Q+Q^{2}\right)(q+Q)\left(q+Q^{2}\right) \neq 0 .
$$

(If $k=0$, we could then find a nilpotent ideal of $H$, namely $\sum_{w \in W} q^{-v(w)} T_{w}$, where $v(w)$ is defined to be the total number of $s_{1}$ 's and $s_{2}$ 's in the canonical expression for $w$ given in Remark 1.5. This would then contradict the fact that $H$ is semisimple.)

In view of the fact that $\left\{f, f s_{2}, f s_{2} s_{1}\right\}$ is a $K$-basis for $f H\left(W_{1}\right)$ (see [4, proof of 5.7]), we get that $f H$ is spanned by $\left\{f T_{w}: w \in S\right\} \cup\left\{s_{2} T_{w}: w \in S\right\} \cup\left\{f s_{2} s_{1} T_{w}: w \in S\right\}$, where $S$ is the set of distinguished right coset representatives of $W_{1}$ in $W$ given in Lemma 1.4.

Suppose now that $x \in W_{2}$. It is easy to see that $T_{x} e=\lambda e$ for some $\lambda \in K$. As a consequence of that, eliminating the elements of $S$ which end in $t_{2}, t$ or $s_{1}$, we get that $f H e$ is spanned by $\left\{f T_{r} e: r \in R\right\}$ where

$$
\begin{aligned}
R= & \left\{1, s_{2}, s_{2} s_{1}, t_{2} t s_{1} s_{2}, s_{2} t_{2} t s_{1} s_{2}, s_{2} s_{1} t_{2} t s_{1} s_{2}, t_{2} t s_{1} t t_{2} s_{2} s_{1} t s_{1} s_{2}, s_{2} t_{2} t s_{1} t t_{2} s_{2} s_{1} t s_{1} s_{2},\right. \\
& \left.s_{2} s_{1} t_{2} t s_{1} t t_{2} s_{2} s_{1} t s_{1} s_{2}\right\} .
\end{aligned}
$$

Consider for example $f T_{r} e$ where $r=t_{2} t s_{1} t t_{2} s_{2} s_{1} t s_{1} s_{2}$. Using the properties of multiplying inside the Hecke algebra, we get (recall $\left.f=x_{7}, e=y_{8}\right)$ :

$$
\begin{aligned}
f T_{r} e= & \left(1+T_{t}\right) T_{s_{1} s_{2}}\left(Q-T_{t}\right)\left(Q q-T_{s_{1} s_{1}}\right)\left(q-T_{s_{1}}\right) T_{r} y_{8} \\
= & \left(q-T_{s_{2}}\right)\left(1+T_{t}\right) T_{s_{1} s_{2}}\left(Q-T_{t}\right)\left(Q q-T_{s_{1} s_{1}}\right) T_{r} y_{8} \quad(\text { see }[4,3.10]) \\
= & \left(q-T_{s_{2}}\right)\left(1+T_{t}\right) T_{s_{1} s_{2}}\left(Q q-T_{s_{2} t s_{1}}\right) T_{r}\left(Q-T_{t}\right) y_{8} \\
& \left(\left(Q-T_{t}\right) \text { commutes with } T_{r} \text { and with }\left(Q q-T_{s_{1} t s_{1}}\right)\right) \\
= & 0
\end{aligned}
$$

since $\left(Q-T_{t}\right) y_{8}=0$. If we now assume that $r=s_{2} t_{2} t s_{1} t t_{2} s_{2} s_{1} t s_{1} s_{2}$ we get that $f T_{r} e=0$ using exactly the same argument as above and noting that $(Q-t)$ commutes with $T_{s_{2}}$ as well.

In fact, using similar argument as in the examples above we can show that $f T_{r} e=0$ for all $r \in R-\left\{s_{2} s_{1} t_{2} t s_{1} s_{2}\right\}$.

Consider now $f T_{r} e$ where $r=s_{2} s_{1} t_{2} t s_{1} s_{2}$. We get,

$$
f T_{s_{2} s_{1} t_{2} s_{1} s_{2}} e=Q^{5} q^{6}(1+Q)(1+q) T_{t_{2} s_{1} s_{2}}+\sum_{\ell(w)>4} \alpha_{w} T_{w}\left(\alpha_{w} \in K\right) .
$$

Since $(1+Q)(1+q) \neq 0$ (we have assumed that $H$ is semisimple), we can conclude that $f T_{s_{2} s_{1} t_{2} s_{1} s_{2}} e \neq 0$. We have thus shown that $f H e$ is 1 -dimensional as a vector over $K$. If we now define $z_{11}=x_{7} T_{s_{2} s_{1} t_{2} t s_{5} s_{2}} y_{8}$, we get that $z_{11} H$ is a simple $H$-module in view of 
Corollary 3.2 . In passing we remark that

$$
z_{11} H=\left((1+q)\left(1+T_{t}\right) T_{s_{1} s_{2}}\left(Q-T_{t}\right)\left(Q q-T_{s_{1} s_{1}}\right) T_{s_{2} s_{1} l_{2} s_{1} s_{2}} y_{8}\right) H .
$$

As a vector space over $K, z_{11} H$ is 9-dimensional and has basis

$\left\{z_{11}, z_{11} s_{2}, z_{11} s_{2} s_{1}, z_{11} s_{2} s_{1} t, z_{11} s_{2} s_{1} t t_{2}, z_{11} s_{2} s_{1} t s_{1}, z_{11} s_{2} s_{1} t s_{1} t_{2}, z_{11} s_{2} s_{1} t s_{1} s_{2}, z_{11} s_{2} s_{1} t s_{1} s_{2} t_{2}\right\}$.

With respect to this basis of $z_{11} H$ we get the following 9-dimensional matrix representation $\sigma$ of $H$. It follows from the discussion above that $\sigma$ is an irreducible representation (provided that $H$ is semisimple). The characters of this representation agree with the characters given in Geck [5]. In [5], Geck calculated the characters of the generic Hecke algebra of type $F_{4}$ by constructing matrix representations of degree at most 8 . (The notation $\{\{$ row 1$\}$, row 2$\}, \ldots$, \{row 9$\}$ to present the matrices is used here.)

$$
\begin{aligned}
\sigma\left(T_{s_{1}}\right)= & \{\{-1,0,0,0,0,0,0,0,0\},\{0,0,1,0,0,0,0,0,0\},\{0, q, q-1,0,0,0,0,0,0\}, \\
& \{0,0,0,0,0,1,0,0,0\},\{0,0,0,0,0,0,1,0,0\},\{0,0,0, q, 0, q-1,0,0,0\}, \\
& \{0,0,0,0, q, 0, q-1,0,0\},\{0,0,0,0,0,0,0,-1,0\},\{0,0,0,0,0,0,0,0,-1\}\}, \\
\sigma\left(T_{s_{2}}\right)= & \{\{0,1,0,0,0,0,0,0,0\},\{q, q-1,0,0,0,0,0,0,0\},\{0,0,-1,0,0,0,0,0,0\}, \\
& \{0,0,0,-1,0,0,0,0,0\},\{0,0,0,0,-1,0,0,0,0\},\{0,0,0,0,0,0,0,1,0\},\{0,0, \\
& 0,0,0,0,0,0,1\},\{0,0,0,0,0, q, 0, q-1,0\},\{0,0,0,0,0,0, q, 0, q-1\}\}, \\
\sigma\left(T_{t}\right)= & \{\{Q, 0,0,0,0,0,0,0,0\},\{0, Q, 0,0,0,0,0,0,0\},\{0,0,0,1,0,0,0,0,0\}, \\
& \{0,0, Q, Q-1,0,0,0,0,0\},\{0,0,0,0, Q, 0,0,0,0\},\{0,0,0,0,0, Q, 0,0,0\}, \\
& \left\{(q-1) q(q+Q),-1+q-q^{2},-1+q,-1+q,-1+q,-1,-1,0,0\right\}, \\
& \{0,0,0,0,0,0,0, Q, 0\},\left\{q\left(-1+q-q^{2}\right),(-1+q)(-1+q+q Q),\right. \\
& 1-q, 1-q, 1-q, 0,0,-1,-1\}\}, \\
\sigma\left(T_{t_{2}}\right)= & \{\{Q, 0,0,0,0,0,0,0,0\},\{0, Q, 0,0,0,0,0,0,0\},\{0,0, Q, 0,0,0,0,0,0\},\{0,0, \\
& 0,0,1,0,0,0,0\},\{0,0,0, Q, Q-1,0,0,0,0\},\{0,0,0,0,0,0,1,0,0\},\{0,0,0, \\
& 0,0, Q, Q-1,0,0\},\{0,0,0,0,0,0,0,0,1\},\{0,0,0,0,0,0,0, Q, Q-1\}\} .
\end{aligned}
$$

3.3. Remarks. (i) We can easily show by checking the set of defining relations for $H$ that the matrices $\sigma\left(T_{s_{1}}\right), \sigma\left(T_{s_{2}}\right), \sigma\left(T_{1}\right), \sigma\left(T_{i_{2}}\right)$ still define a representation of $H$ even in the case when no restrictions on $q, Q$ are made. (Hence, if $H$ is not semisimple, $\sigma$ is still a representation of $H$ through not necessarily an irreducible one.)

(ii) Using the automorphism $\theta$ of $H$ given in 2.3 we can get another 9-dimensional matrix representation of $H$ from the one given above.

(iii) In the special case $q=Q=1$, char $K=0$, the character of the matrix representation $\sigma$ corresponding to $z_{11} H$ is $\chi_{11}$. This is expected from the way $f$ and $e$ were chosen (compare Remark 2.6 (the case $i=7, j=8$ )).

3.4. The results (compare Remarks 2.5 and 2.6). With suitable choices of $f$ and $e$, and considering $f H e$, generators $z_{m}$ (see the table below) were constructed $(10 \leq m \leq$ $13,21 \leq m \leq 24$ ) such that, provided that $H$ is semisimple, the right ideal $z_{m} H$ is a simple $H$-module.

The proof that $z_{m} H$ is simple is very similar to the proof given above for the case $m=11$. Note that for the choices of $f$ and $e$ given below, $e^{2}=k e$ for some $k \in K, k \neq 0$, and $f H e$ was proved to be 1-dimensional as a vector space over $K$ (compare Corollary 3.2 and the case $m=11$ ). 
THE HECKE ALGEBRA OF TYPE $F_{4}$

\begin{tabular}{|c|c|c|}
\hline$e$ & $f$ & Basis of $f H e$ as a vector space over $K$ \\
\hline$y_{1}$ & $x_{6}$ & $x_{6} T_{s_{2} s_{1} t_{2} s_{5} s_{2}} y_{1}=z_{10}$ \\
$y_{8}$ & $x_{7}$ & $x_{7} T_{s_{2} s_{1} t_{2} t_{1} s_{2}} y_{8}=z_{11}$ \\
$x_{8}$ & $y_{7}$ & $y_{7} T_{t_{2} s_{2} s_{1} t t_{2}} x_{8}=z_{12}$ \\
$y_{10}$ & $x_{5}$ & $x_{5} T_{t_{2} t s_{1} s_{2}} y_{10}=z_{13}$ \\
$y_{8}$ & $x_{4}$ & $x_{4} T_{s_{1} t_{2} s_{1} s_{2}} y_{8}=z_{21}$ \\
$y_{3}$ & $x_{7}$ & $x_{7} T_{t_{2} s_{1}, s_{2}} y_{3}=z_{22}$ \\
$x_{8}$ & $y_{4}$ & $y_{4} T_{t s_{2} s_{1} t t_{2}} x_{8}=z_{23}$ \\
$x_{3}$ & $y_{7}$ & $y_{7} T_{s_{2} s_{1} t t_{2}} x_{3}=z_{24}$ \\
\hline
\end{tabular}

In the special case char $K=0, Q=q=1, z_{m} H$ gives a representation of $W$ with character $\chi_{m}(10 \leq m \leq 13,21 \leq m \leq 24)$. (Compare Remark 2.6.)

From these right ideals matrix representations of $H(W)$ can be constructed by fixing a $K$-basis for $z_{m} H$ as in the example given above for $m=11$. For example, for the case $m=24$, we get the following 8-dimensional matrix representation $\tau$ of $H(W)$ :

$$
\begin{aligned}
\tau\left(T_{s_{1}}\right)=\{ & \{-1,0,0,0,0,0,0,0\},\{0,-1,0,0,0,0,0,0\},\{0,0,0,1,0,0,0,0\},\{0,0, q, \\
& q-1,0,0,0,0\},\{0,0,0,0,-1,0,0,0\},\{0,0,0,0,0,-1,0,0\},\{0,0,0,0,0, \\
& 0,0,1\},\{0,0,0,0,0,0, q,-1+q\}\}, \\
\tau\left(T_{s_{2}}\right)=\{ & \{-1,0,0,0,0,0,0,0\},\{0,-1,0,0,0,0,0,0\},\{0,0,-1,0,0,0,0,0\},\{0,0,0, \\
& 0,1,0,0,0\},\{0,0,0, q, q-1,0,0,0\},\{0,0,0,0,0,0,1,0\},\{0,0,0,0,0, q, \\
& q-1,0\},\{0,0,0,0,0,0,0,-1\}\}, \\
\tau\left(T_{t}\right)= & \{\{Q, 0,0,0,0,0,0,0\},\{0,0,1,0,0,0,0,0\},\{0, Q,-1+Q, 0,0,0,0,0\},\{0,0, \\
& 0,0,0,1,0,0\},\{0,0,0,0,0,0,1,0\},\{0,0,0, Q, 0,-1+Q, 0,0\},\{0,0,0,0, \\
& \left.Q, 0,-1+Q, 0\},\left\{q^{3}+q Q+q^{2} Q-q^{3} Q^{2}, q^{3}, q^{3},-q^{2}, q,-q^{2}, q,-1\right\}\right\}, \\
\tau\left(T_{t_{2}}\right)= & \{\{0,1,0,0,0,0,0,0\},\{Q,-1+Q, 0,0,0,0,0,0\},\{0,0, Q, 0,0,0,0,0\}, \\
& \{0,0,0, Q, 0,0,0,0\},\{0,0,0,0, Q, 0,0,0\},\{q, q, q,-1,0,-1,0,0\}, \\
& \{-q,-q,-q, 0,-1,0,-1,0\},\{q, q, 0,-q, 1,0,0,-1\}\},
\end{aligned}
$$

3.5. Remarks. (i) Again we can easily show by checking the set of defining relations for $H$ that $\tau$ is still a representation of $H$ even when no restrictions on $q$ or $Q$ are being made.

(ii) Note that the representing matrices of $\tau$ for the generators involve no denominators. (Compare [5, p. 66] where the representing matrices of the corresponding representation involve denominators.)

(iii) Comparing the expressions for $z_{22}$ and $z_{24}$ we see that $z_{22}$ can be obtained from $z_{24}$ by interchanging $T_{s_{1}}$ with $T_{c}, T_{s_{2}}$ with $T_{s_{2}}$, and also interchanging the roles of $Q$ and $q$. Thus a matrix representation for $z_{22} H$ can be obtained directly from $\tau$ in the obvious way. (Compare Remark 1.1 and [5, p. 67].) In the same way we can obtain $z_{23}$ from $z_{21}$ and $z_{12}$ from $z_{11}$.

Finally we consider the case $i=6, j=9$ in the table in Remark 2.6. Now $x_{6} H\left(W_{1}\right)$ is 3-dimensional and $y_{9} H\left(W_{2}\right)$ is 2-dimensional as vector spaces over $K$, so it is not necessarily true that either of $x_{6}^{2}=k x_{6}$ or $y_{9}^{2}=k y_{9}$ holds. However, if $H$ is semisimple, 
$y_{9} H=\varepsilon H$ for some idempotent $\varepsilon$. Thus, considering $x_{6} H \varepsilon$ as a vector space over $K$, we expect to be able to construct a 16-dimensional representation of $H$ which, upon specializing $q, Q$ to 1 has character $\chi_{25}$.

ACKNowledgement. I would like to acknowledge some useful discussions on this subject with Professor G. D. James.

\section{REFERENCES}

1. R. W. Carter, Finite groups of Lie type. Conjugacy classes and complex characters (Wiley, 1985).

2. C. W. Curtis and I. Reiner, Methods of representation theory, vols I, II (Wiley, 1981/1987).

3. R. Dipper and G. James, Representations of Hecke algebras of general linear groups, Proc. London Math. Soc. (3) 52 (1986), 20-52.

4. R. Dipper and G. James, Representations of Hecke algebras of type $B_{n}$, J. Algebra 146 (1992), 454-481.

5. M. Geck, On the character values of Iwahori-Hecke algebras of exceptional type, Proc. London Math. Soc. (3) 68 (1994), 51-76.

6. C. Pallikaros, Representations of Hecke algebras of type $D_{n}$, J. Algebra 169 (1994), 20-48.

Department of Mathematics and Statistics

UNIVERSITY OF CYPRUS

P.O. Box 537

1678 Nicosia

CYPRUS 